\title{
Experimentally accessible signatures of Auger scattering in graphene
}

\author{
Torben Winzer, ${ }^{1}$ Roland Jago, ${ }^{2}$ and Ermin Malic ${ }^{2, *}$ \\ ${ }^{1}$ Institut für Theoretische Physik, Technische Universität Berlin, Hardenbergstr. 36, 10623 Berlin, Germany \\ ${ }^{2}$ Chalmers University of Technology, Department of Physics, SE-412 96 Gothenburg, Sweden \\ (Received 3 August 2016; revised manuscript received 29 November 2016; published 23 December 2016)
}

\begin{abstract}
The gapless and linear electronic band structure of graphene opens up Auger scattering channels bridging the valence and the conduction band and changing the charge carrier density. Here, we reveal experimentally accessible signatures of Auger scattering in optically excited graphene. To be able to focus on signatures of Auger scattering, we apply a low excitation energy, weak pump fluences, and a cryostatic temperature, so that all relevant processes lie energetically below the optical phonon threshold. In this regime, carrier-phonon scattering is strongly suppressed and Coulomb processes govern the carrier dynamics. Depending on the excitation regime, we find an accumulation or depletion of the carrier occupation close to the Dirac point. This reflects well the behavior predicted from Auger-dominated carrier dynamics. Based on this observation, we propose a multicolor pump-probe experiment to uncover the extreme importance of Auger channels for the nonequilibrium dynamics in graphene.
\end{abstract}

DOI: 10.1103/PhysRevB.94.235430

A remarkable consequence of the unique electronic band structure of graphene is the possibility of carrier multiplication (CM), i.e., generation of multiple electron-hole pairs through internal Auger scattering [1]. These processes are specific Coulomb-driven scattering events, where one electron bridges the valence and the conduction band, while the other involved electron remains in the same band, cf. Figs. 1(a) and 1(c). As a result, Auger scattering changes the overall charge carrier density, i.e., the number of electrons in the conduction band and the number of holes in the valence band. We distinguish Auger recombination (AR) and the inverse process of impact excitation (IE). The latter leads to an increase of the carrier density and is responsible for the appearance of CM. In previous studies, a significant carrier multiplication has been predicted in optically excited graphene [2-4] as well as Landau-quantized graphene [5]. Meanwhile carrier multiplication has also been demonstrated in time-dependent ARPES measurements [6] as well as in high-resolution pumpprobe studies [7-9]. In doped graphene samples, a so-called hot CM has been theoretically predicted [10] and experimentally measured [11,12]. Here, CM occurs via Coulomb-induced intraband scattering bridging the states below and above the Fermi level, as sketched in Fig. 1(a).

In this paper, we propose a strategy to directly reveal the presence of strongly efficient Auger scattering in graphene. Our work is motivated by a very recent experimental study performed by J. Koenig-Otto and co-workers [13], which demonstrated that phonon-driven carrier relaxation can be strongly suppressed by applying low excitation energies, weak pump fluences, and cryostatic temperatures. This allows a study of purely Coulomb-induced carrier dynamics. Furthermore, the authors found that collinear Coulomb scattering leads to an ultrafast carrier thermalization on a femtosecond timescale, whereas noncollinear Coulomb scattering is remarkably slow giving rise to a picosecond decay of the initial optically induced carrier anisotropy. The idea of the current work is now to exploit these insights to visualize the

\footnotetext{
*ermin.malic@chalmers.se
}

impact of collinear Coulomb channels (Auger scattering) and to present a recipe for their experimental demonstration. Based on microscopic calculations, we show how impact excitation accumulates carriers close to the Dirac point, whereas Auger recombination depletes the carrier population in this region. We present a recipe of how to exploit this behavior as a clear signature to identify Auger scattering in multicolor pump-probe measurements.

To microscopically model the dynamics of optically excited carriers in graphene, we have developed a theoretical approach based on the density matrix formalism [14-17]. The core of the approach is graphene Bloch equations allowing us to temporally and spectrally resolve the dynamics of electrons and phonons in different regimes [18,19]. We take into account all relevant many-particle interactions on a consistent microscopic footing including carrier-light, carrier-carrier, and carrier-phonon interaction. The coupled system of differential equations for (i) the occupation probability $\rho_{\mathbf{k}}^{\lambda}$ in the state $(\lambda, \mathbf{k})$ with the electronic momentum $\mathbf{k}$ and the band $(\lambda=c, v)$, (ii) the microscopic polarization $p_{\mathbf{k}}$ as a measure for the optically induced transition probability between the valence (v) and conduction (c) band, and (iii) the phonon occupation $n_{\mathbf{q}}^{j}$ in the state $(j, \mathbf{q})$ with the phonon momentum $\mathbf{q}$ and different optical and acoustic modes $j$ reads in second-order Born-Markov approximation [19]:

$$
\begin{aligned}
& \dot{\rho}_{\mathbf{k}}^{\lambda}=2 \Im\left[\Omega_{\mathbf{k}}^{v c *} p_{\mathbf{k}}\right]+\left.\dot{\rho}_{\mathbf{k}}^{\lambda}\right|_{\text {scat }}, \\
& \dot{p}_{\mathbf{k}}=\left[i \Delta \omega_{\mathbf{k}}-\gamma_{\mathbf{k}}\right] p_{\mathbf{k}}-i \Omega_{\mathbf{k}}^{v c}\left[\rho_{\mathbf{k}}^{c}-\rho_{\mathbf{k}}^{v}\right]+\mathcal{U}_{\mathbf{k}}, \\
& \dot{n}_{\mathbf{q}}^{j}=-\gamma_{p h}\left[n_{\mathbf{q}}^{j}-n_{B}\right]+\Gamma_{j, \mathbf{q}}^{e m}\left[1+n_{\mathbf{q}}^{j}\right]-\Gamma_{j, \mathbf{q}}^{\mathrm{abs}} n_{\mathbf{q}}^{j} .
\end{aligned}
$$

The optical excitation and the generation of a nonequilibrium carrier distribution are determined by the Rabi frequency $\Omega_{\mathbf{k}}^{v c}(t)$. The electronic transition frequency is denoted by $\Delta \omega_{\mathbf{k}}=\left(\omega_{\mathbf{k}}^{v}-\omega_{\mathbf{k}}^{c}\right)$ and leads to an oscillation of the microscopic polarization $p_{\mathbf{k}}(t)$, which is damped by the diagonal $\gamma_{\mathbf{k}}(t)$ and the off-diagonal dephasing $\mathcal{U}_{\mathbf{k}}(t)$. For phonons, we assume the Bose-Einstein distribution $n_{B}$ as the initial condition for the phonon occupation and include a phenomenological phonon decay rate $\gamma_{p h}$ [20]. The manyparticle interactions are expressed by phonon emission $\Gamma_{j, \mathbf{q}}^{e m}(t)$ 

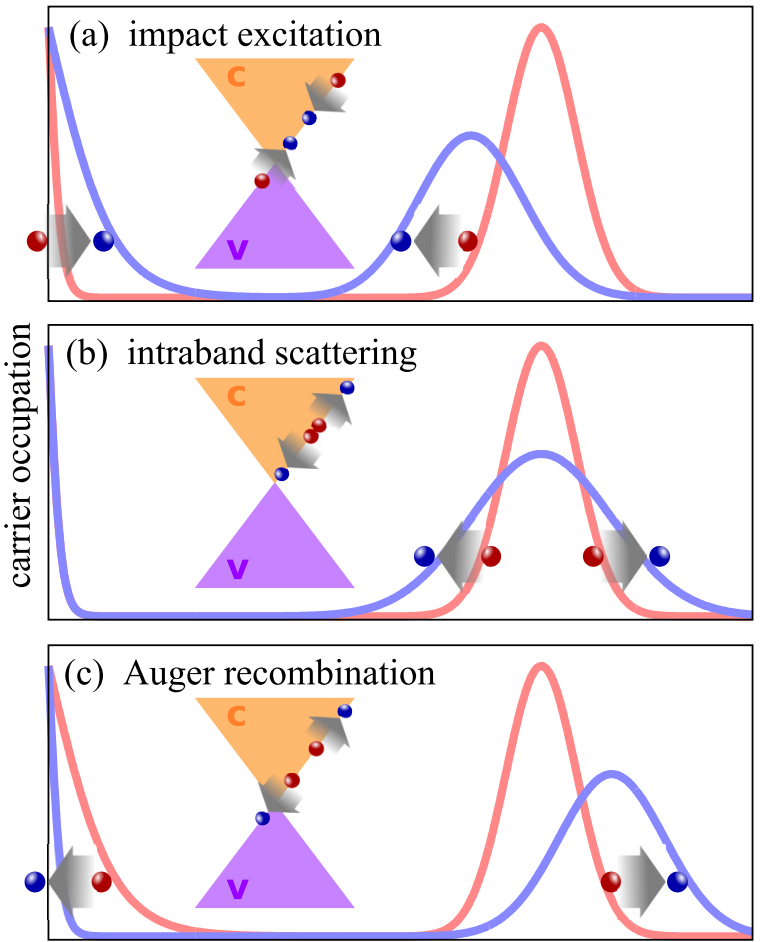

carrier energy

FIG. 1. Schematic illustration of Coulomb scattering processes. (a) Impact excitation gives rise to an accumulation of carriers close to the Dirac point and a redshift of the optically excited carrier occupation, (b) Coulomb-induced intraband scattering accounts for a spectral broadening around the excitation energy, and (c) Auger recombination reduces the low-energy carrier occupation and leads to a blueshift of the excited carriers.

and absorption rates $\Gamma_{j, \mathbf{q}}^{\mathrm{abs}}(t)$. The exact expression can be found in Refs. [18,19].

The carrier relaxation dynamics is determined within the second-order Born-Markov approximation resulting in a Boltzmann-like scattering equation $[3,18,19,21]$ :

$$
\left.\dot{\rho}_{\mathbf{k}}^{\lambda}\right|_{\text {scat }}=\sum_{\lambda_{1}, \mathbf{k}^{\prime}} \mathcal{W}_{\mathbf{k}^{\prime} \rightarrow \mathbf{k}}^{\lambda_{1} \rightarrow \lambda} \rho_{\mathbf{k}^{\prime}}^{\lambda_{1}}\left(1-\rho_{\mathbf{k}}^{\lambda}\right)-\mathcal{W}_{\mathbf{k}^{\prime} \leftarrow \mathbf{k}}^{\lambda_{1} \leftarrow \lambda}\left(1-\rho_{\mathbf{k}^{\prime}}^{\lambda_{1}}\right) \rho_{\mathbf{k}}^{\lambda}
$$

including Coulomb- and phonon-induced scattering processes. We include collinear and noncollinear carrier-carrier scattering as well as scattering with optical and acoustic phonons. In previous studies, we have revealed that the impact of acoustic phonons is negligibly small. The corresponding scattering time is in the range of a few hundreds of picoseconds and only contributes to the cooling of excited carriers at very late times $[18,22]$. Note however that in disordered graphene samples, defect-assisted scattering with acoustic phonons might significantly increase the available phase space, and these so-called supercollisions can become an important scattering channel close to the Dirac point [23].

In the case of the Coulomb interaction, the probability to scatter from the state $\left(\lambda_{1}, \mathbf{k}^{\prime}\right)$ to $(\lambda, \mathbf{k})$ is given by:

$$
\mathcal{W}_{\mathbf{k}^{\prime} \rightarrow \mathbf{k}}^{\lambda_{1} \rightarrow \lambda}=\sum_{\lambda_{2}, \lambda_{3}, \mathbf{q}} \mathcal{V}_{\mathbf{k}, \mathbf{k}^{\prime}, \mathbf{q}}^{\lambda, \lambda_{2}, \lambda_{3}, \lambda_{3}}\left(1-\rho_{\mathbf{k}^{\prime}+\mathbf{q}}^{\lambda_{3}}\right) \rho_{\mathbf{k}+\mathbf{q}}^{\lambda_{2}},
$$

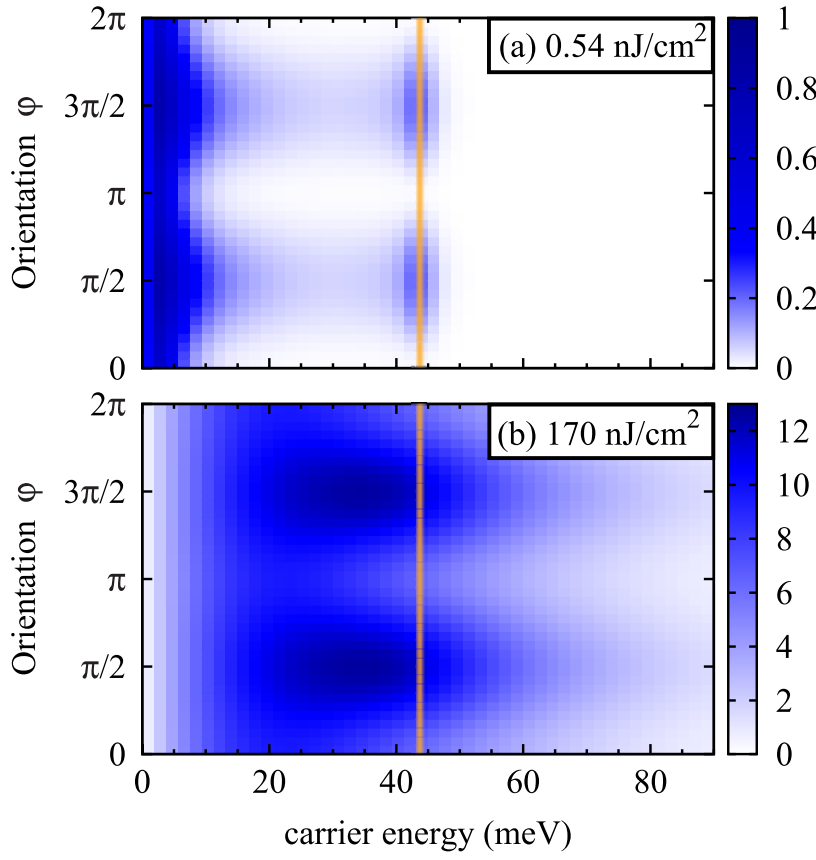

FIG. 2. Signatures of Auger processes. Carrier occupation $\rho_{k, \varphi}$ weighted by the density of states (DOS) is shown as a function of carrier energy and angle $\varphi$ at the maximum of the excitation pulse ( $t=0 \mathrm{fs}$ ) for (a) a low pump fluence of $0.54 \mathrm{~nJ} / \mathrm{cm}^{2}$ and (b) a higher pump fluence of $170 \mathrm{~nJ} / \mathrm{cm}^{2}$. A qualitatively different behavior is observed: While in the low excitation regime, the carriers accumulate at low energies close to the Dirac point (reflecting impact excitation); at higher pump fluences we observe a strong spectral broadening of the carrier distribution including an asymmetric smearing towards higher energies (reflecting Auger recombination). The orange-shaded vertical area corresponds to the spectral width of the excitation pulse.

where $\mathcal{V}_{\mathbf{k}, \mathbf{k}^{\prime}, \mathbf{q}}^{\lambda, \lambda_{2}, \lambda_{3}}$ contains the screened Coulomb matrix element as well as a delta-function ensuring energy conservation. The simultaneous conservation of momentum (through the matrix element) and energy restricts Auger processes to parallel scattering along the Dirac cone [24], cf. the insets of Fig. 1.

To study experimentally accessible signatures of Auger processes on the basis of their impact on the spectral distribution of the excited carriers, we consider an excitation regime, where the dynamics is approximately fully governed by Coulomb-induced scattering. This is achieved by applying a cryostatic temperature of $16 \mathrm{~K}$, an excitation energy of $88 \mathrm{meV}$, and low pump fluences in the range of $\mathrm{nJ} / \mathrm{cm}^{2}$, so that all relevant processes lie energetically below the optical phonon threshold $(\propto 200 \mathrm{meV})$ [13]. Recently, it has been demonstrated that under these conditions carrier-phonon scattering is strongly suppressed resulting in a long-lived anisotropic carrier distribution after excitation with linearly polarized light [13].

Figure 2 illustrates the carrier distribution as a function of carrier energy $\varepsilon=\hbar v_{F}|\mathbf{k}|$ and angle $\varphi$, where $v_{F}$ is the Fermi velocity. To account for the available phase space and the real number of present carriers, the carrier occupation $\rho_{k, \varphi}$ has been weighted with the density of states that is proportional to $|\mathbf{k}|$ in graphene. The carrier distribution is shown at the maximum of the excitation pulse ( $t=0 \mathrm{fs}$ ) for (a) a low pump fluence of 
$0.54 \mathrm{~nJ} / \mathrm{cm}^{2}$ and (b) a higher pump fluence of $170 \mathrm{~nJ} / \mathrm{cm}^{2}$. For both excitation strengths, the excited anisotropy of the carrier distribution due to the linear polarization of the excitation pulse $[25,26]$ is conserved on the timescale of the pulse (FWHM of $4 \mathrm{ps)}$ [13]. However, the energetic distribution strongly depends on the excitation strength and exhibits qualitative differences between the low and the strong excitation regime. At low pump fluences [Fig. 2(a)], we clearly identify the predominant role of impact excitation: The excited carriers scatter to energetically lower states, which gives rise to the generation of secondary carriers in the region close to the Dirac point, cf. also the schematic sketch in Fig. 1(a). In particular, no carriers are found above the excitation energy. This is in sharp contrast to the behavior at the higher fluence [Fig. 2(b)], where the excited carriers scatter to both energetically lower and higher states. Collinear Coulomb-induced intraband scattering is mainly responsible for this spectral broadening, cf. Fig. 1(b). Additionally, an asymmetric smearing of carrier occupation towards higher energies as well as the absence of carriers in the region close to the Dirac point can be observed, which are clear signatures for efficient Auger recombination, cf. Fig. 1(c). Note that AR also contributes to a spectral broadening even at the low pump fluence, $\mathrm{cf}$. the orange-shaded vertical area denoting the spectral width of the excitation pulse.

To get further insights, we study the time-dependent Auger scattering rates $\gamma_{I E}(t)$ and $\gamma_{A R}(t)$ revealing how fast carriers are generated via impact excitation and annihilated via Auger recombination. The rates are defined by the integration of the Boltzmann-like equation [Eq. (2)] over all processes that increase or decrease the carrier density, respectively:

$$
\gamma_{I E}=\left.\sigma_{S} \sigma_{\mathcal{V}} \sum_{\lambda \mathbf{k}} \dot{\rho}_{\mathbf{k}}^{\lambda}\right|_{I E} \quad \text { and } \quad \gamma_{A R}=\left.\sigma_{S} \sigma_{\mathcal{V}} \sum_{\lambda \mathbf{k}} \dot{\rho}_{\mathbf{k}}^{\lambda}\right|_{A R},
$$

where $\sigma_{s}\left(\sigma_{\mathcal{V}}\right)$ accounts for the degeneracy of spin (valley). In the low-excitation regime, we clearly observe an asymmetry between both Auger rates in favor of impact excitation $\gamma_{I E}$, cf. Fig. 3(a). Note that this asymmetry corresponds to a net carrier multiplication [2], which is accomplished when both rates become equal, i.e., after approximately $10 \mathrm{ps}$. For the higher pump fluence of $170 \mathrm{~nJ} / \mathrm{cm}^{2}$, impact excitation $\gamma_{I E}$ is initially still the dominant Auger scattering channel. However, already before the center of the excitation pulse is reached (at $0 \mathrm{ps),} \mathrm{Auger} \mathrm{recombination} \gamma_{A R}$ overtakes $\gamma_{I E}$ [Fig. 3(b)] corresponding to a Coulomb-induced loss of charge carriers.

So far we have found that impact excitation accumulates carriers close to the Dirac point and is the predominant channel in the low-excitation regime, while Auger recombination determines the dynamics at higher pump fluences giving rise to a depletion of the carrier occupation close to the Dirac point and leading to an asymmetric smearing of the carrier distribution towards energies above the optical excitation. This characteristic behavior can be exploited to experimentally identify the presence of extremely efficient Auger scattering in optically excited graphene. A straightforward way to access this characteristic behavior is to perform time-resolved ARPES measurements at different excitation regimes and for an excitation energy clearly below the optical phonon energy. A different approach is to perform a multicolor pump-probe experiment, where the probe pulse scans the carrier distribution

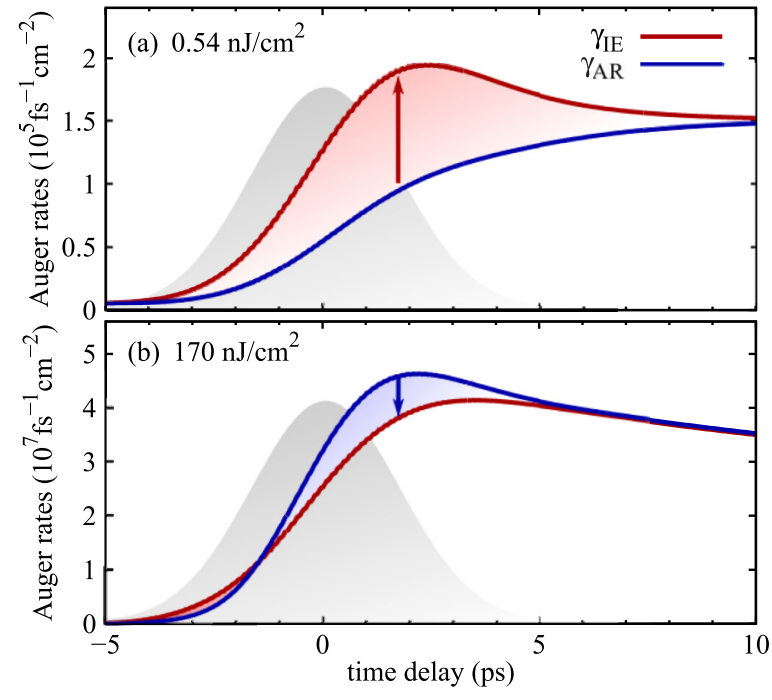

FIG. 3. Auger scattering rates in different excitation regimes. The rates for impact excitation $\gamma_{I E}$ and Auger recombination $\gamma_{A R}$ are shown for (a) $0.54 \mathrm{~nJ} / \mathrm{cm}^{2}$ and (b) $170 \mathrm{~nJ} / \mathrm{cm}^{2}$. In the low excitation regime, there is a clear asymmetry in favor of $\gamma_{I E}$, while for higher pump fluences $\gamma_{A R}$ overtakes impact excitation. The Gaussian excitation pulse is depicted in the background.

close to the Dirac point and in the spectral region above the optical excitation.

To investigate Auger features in multicolor pump-probe experiments, we have calculated differential transmission spectra (DTS) in the strong AR-dominated and the weak IE-dominated excitation regime. We find clear signatures of Auger processes in the DTS amplitude and the DTS decay as a function of the probe energy: At low pump fluence, we find that the amplitude is drastically reduced with the increasing probe energy, cf. Fig. 4(a). It decreases by about a factor of 50, when moving the probe energy from $20 \mathrm{meV}$ (red line, below the optical excitation energy) to $88 \mathrm{meV}$ (green line, monochromatic) and it vanishes (within the numerical accuracy) at $118 \mathrm{meV}$ (blue line, above the excitation energy). This reflects well the carrier occupation illustrated in Fig. 2(a), where impact excitation accumulates carriers close to the Dirac point and where no carriers scatter to states above the excitation energy. The situation is qualitatively different in the higher-excitation regime, where the reduction of the amplitude is much smaller and ranges within one order of magnitude, cf. Fig. 4(b). This behavior directly reflects the spectral broadening of the carrier distribution at high fluences, where one can find carriers below and above the excitation energy, cf. Fig. 2(b).

Besides the change in the differential transmission amplitude, there is also a significant difference regarding the DTS decay depending on the probe energy and the excitation regime. Since at low pump fluences the optically excited carriers quickly leave the energy range of the excitation by scattering into energetically lower states via impact excitation, the monochromatic DTS decays very fast and at the same time the DTS at $20 \mathrm{meV}$ quickly increases, cf. the green and the red line in Fig. 4(a). In contrast, at higher pump fluences, we observe a very slow DTS decay for all three 


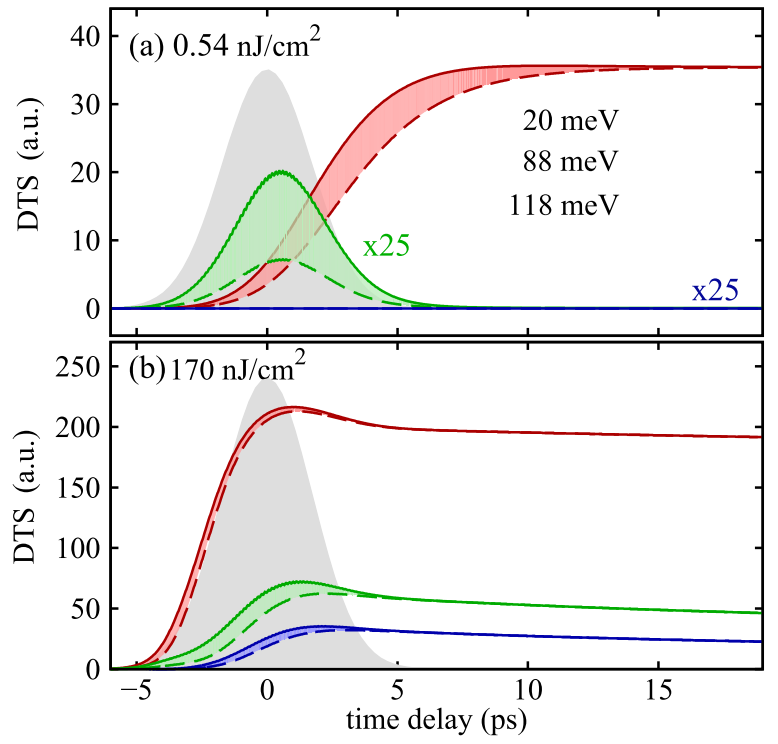

FIG. 4. Experimentally accessible signatures of Auger processes. Differential transmission spectra (DTS) in the (a) weak and (b) higher excitation regime. The pump energy is fixed to $88 \mathrm{meV}$, while the probe energy is varied ( $20 \mathrm{meV}$ red, $88 \mathrm{meV}$ green, and $118 \mathrm{meV}$ blue). We observe in the weak excitation regime a drastic decrease in the DTS amplitude as the probe pulse increases. This reflects the IE-dominated carrier dynamics resulting in a carrier accumulation close to the Dirac point and a vanishing carrier population in the states above the excitation energy [cf. Fig. 2(a)]. The change of the amplitude is much smaller in the AR-dominated higher excitation regime. This reflects the large spectral broadening of the carrier distribution covering energies below and above the excitation energy [cf. Fig. 2(b)]. The solid and dashed lines show the parallel and the perpendicular polarization of the pump and the probe pulse, respectively.

probe pulses. Here, the carrier thermalization occurs already on the timescale of the optical pump pulse and the redistribution of carriers via Auger recombination and Coulomb-induced intraband scattering is very fast [cf. also Fig. 3(b)]. As a result, the decay of differential transmission can only be induced by carrier-phonon scattering. However, since the scattering with optical phonons is strongly suppressed due to the excitation energy well below the phonon energy and since acoustic phonons are very week in graphene [18,22], the DTS decays on a very slow timescale.
Figure 4 also illustrates the lifetime of the anisotropic carrier distribution at different probe energies and excitation regimes. The DTS is shown for parallel (solid line) and perpendicular (dashed) polarization of the pump and the probe pulse. As long as there is a difference in DTS, an anisotropic carrier distribution is present. In agreement with Ref. [13], we find that the anisotropy is much more pronounced in the low-excitation regime, where it persists on a timescale of 10 ps, cf. Fig. 4(a). In contrast, at higher pump fluences, the carrier distribution becomes isotropic much faster and the DTS curves for different relative polarizations become indistinguishable already during the excitation pulse, cf. Fig. 4(b). Since the carrier-phonon interaction is strongly suppressed at the investigated excitation energy, noncollinear Coulomb processes determine the scattering across the Dirac cone that reduces the anisotropy in the optically excited carrier distribution. As a result, the larger the fluence, the faster can an isotropic carrier distribution be reached. Finally, we also observe a less pronounced anisotropy when probing below or above the excitation energy (compared to the monochromatic case). This can be ascribed to the reduction of the anisotropy during the scattering events bringing optically excited carriers to energetically lower or higher states.

In summary, we have presented a microscopic study of the impact of Auger processes on the spectral distribution of optically excited carriers. We focus on a regime characterized by a cryostatic temperature and a low excitation energy, where carrier-phonon scattering is strongly suppressed and Coulomb processes govern the carrier dynamics. Depending on the excitation regime, Auger recombination or the inverse process of impact excitation dominates the carrier dynamics. While $\mathrm{AR}$ and Coulomb-induced intraband scattering leads to a spectrally broadened carrier distribution around the excitation energy, IE gives rise to an accumulation of carriers close to the Dirac point. Based on these insights, we propose a multicolor pump-probe experiment for a direct demonstration of Auger channels in graphene.

This project has received funding from the European Union's Horizon 2020 research and innovation programme under Grant agreement No. 696656. Furthermore, we gratefully acknowledge support from the Swedish Research Council (VR) and the Deutsche Forschungsgemeinschaft (DFG) through SPP 1459 and SFB 658. Finally, we thank Andreas Knorr (TU Berlin) for valuable and inspiring discussions.
[1] A. Nozik, Physica E 14, 115 (2002).

[2] T. Winzer, A. Knorr, and E. Malic, Nano Lett. 10, 4839 (2010).

[3] T. Winzer and E. Malić, Phys. Rev. B 85, 241404 (2012).

[4] A. Tomadin, D. Brida, G. Cerullo, A. C. Ferrari, and M. Polini, Phys. Rev. B 88, 035430 (2013).

[5] F. Wendler, A. Knorr, and E. Malic, Nat. Commun. 5, 3703 (2014).

[6] I. Gierz, F. Calegari, S. Aeschlimann, M. C. Cervantes, C. Cacho, R. T. Chapman, E. Springate, S. Link, U. Starke, C. R. Ast, and A. Cavalleri, Phys. Rev. Lett. 115, 086803 (2015).
[7] T. Plötzing, T. Winzer, E. Malic, D. Neumaier, A. Knorr, and H. Kurz, Nano Lett. 14, 5371 (2014).

[8] M. Mittendorff, F. Wendler, E. Malic, A. Knorr, M. Orlita, M. Potemski, C. Berger, W. A. de Heer, H. Schneider, M. Helm, and S. Winnerl, Nat. Phys. 11, 75 (2015).

[9] D. Brida, A. Tomadin, C. Manzoni, Y. J. Kim, A. Lombardo, S. Milana, R. R. Nair, K. S. Novoselov, A. C. Ferrari, G. Cerullo, and M. Polini, Nat. Commun. 4, 1987 (2013).

[10] F. Kadi, T. Winzer, A. Knorr, and E. Malic, Sci. Rep. 5, 16841 (2015). 
[11] J. C. Johannsen, S. Ulstrup, A. Crepaldi, F. Cilento, M. Zacchigna, J. A. Miwa, C. Cacho, R. T. Chapman, E. Springate, F. Fromm, C. Raidel, T. Seyller, P. D. C. King, F. Parmigiani, M. Grioni, and P. Hofmann, Nano Lett. 15, 326 (2015).

[12] K. J. Tielrooij, J. C. W. Song, S. A. Jensen, A. Centeno, A. Pesquera, A. Zurutuza Elorza, M. Bonn, L. S. Levitov, and F. H. L. Koppens, Nat. Phys. 9, 248 (2013).

[13] J. C. Koenig-Otto, M. Mittendorff, T. Winzer, E. Malic, A. Knorr, C. Berger, W. A. de Heer, A. Pashkin, H. Schneider, M. Helm, and S. Winnerl1, Phys. Rev. Lett. 117, 087401 (2016).

[14] H. Haug and S. W. Koch, Quantum Theory of the Optical and Electronic Properties of Semiconductors (World Scientific, Singapore, 2004).

[15] F. Rossi and T. Kuhn, Rev. Mod. Phys. 74, 895 (2002).

[16] M. Kira and S. Koch, Prog. Quantum Electron. 30, 155 (2006).

[17] A. Knorr, S. Hughes, T. Stroucken, and S. W. Koch, Chem. Phys. 210, 27 (1996).

[18] E. Malic, T. Winzer, E. Bobkin, and A. Knorr, Phys. Rev. B 84, 205406 (2011).
[19] E. Malic and A. Knorr, Graphene and Carbon Nanotubes: Ultrafast Optics and Relaxation Dynamics, 1st ed. (Wiley-VCH, Berlin, 2013).

[20] K. Kang, D. Abdula, D. G. Cahill, and M. Shim, Phys. Rev. B 81, 165405 (2010).

[21] U. Briskot, M. Schuett, I. V. Gornyi, M. Titov, B. N. Narozhny, and A. D. Mirlin, Phys. Rev. B 92, 115426 (2015).

[22] S. Winnerl, M. Orlita, P. Plochocka, P. Kossacki, M. Potemski, T. Winzer, E. Malic, A. Knorr, M. Sprinkle, C. Berger, W. A. de Heer, H. Schneider, and M. Helm, Phys. Rev. Lett. 107, 237401 (2011).

[23] J. C. W. Song, M. Y. Reizer, and L. S. Levitov, Phys. Rev. Lett. 109, 106602 (2012).

[24] F. Rana, Phys. Rev. B 76, 155431 (2007).

[25] E. Malic, T. Winzer, and A. Knorr, Appl. Phys. Lett. 101, 213110 (2012).

[26] A. Grüneis, R. Saito, G. G. Samsonidze, T. Kimura, M. A. Pimenta, A. Jorio, A. G. Souza Filho, G. Dresselhaus, and M. S. Dresselhaus, Phys. Rev. B 67, 165402 (2003). 